\title{
Giant lipoma of hand with carpal tunnel syndrome
}

\author{
Murat Erdoğan*, Alper Çıraklı, Eyüp Çağatay Zengin, Hicabi Sezgin, Ahmet Pişkin \\ Department of Orthopedics and Traumatology, Faculty of Medicine, Ondokuz Mayis University
}

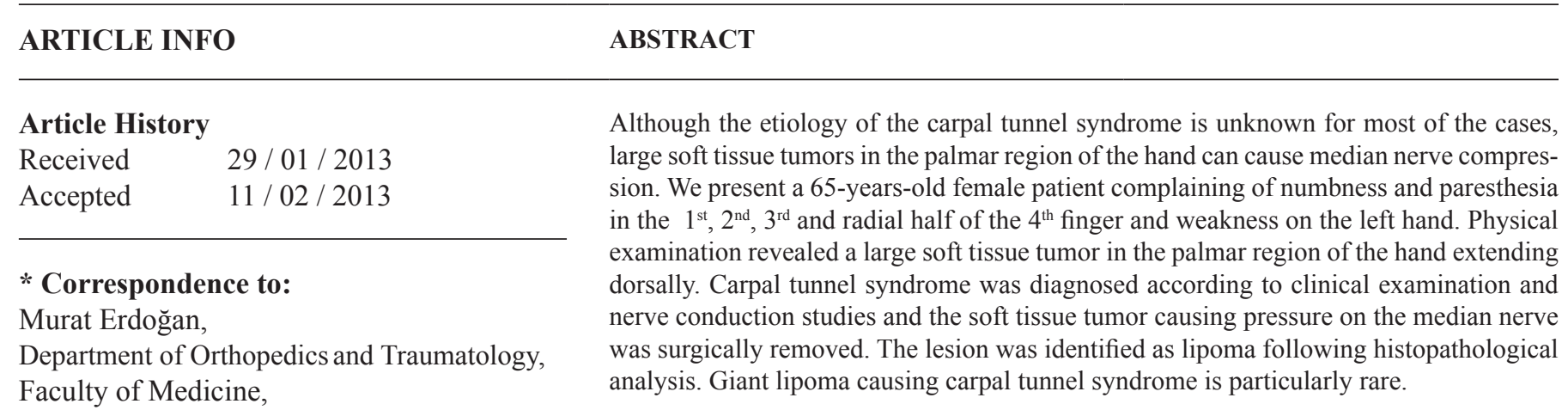

Ondokuz Mayis University, Samsun, Turkey

e-mail: drmuraterdogan@hotmail.com

\section{Keywords:}

Carpal tunnel syndrome

Giant lipoma

Hand

Treatment

Tumour

J. Exp. Clin. Med., 2013; 30:179-180

\begin{abstract}
Although the etiology of the carpal tunnel syndrome is unknown for most of the cases, in the $1^{\text {st }}, 2^{\text {nd }}, 3^{\text {rd }}$ and rat examination revealed a large soft tissue tumor in the palmar region of the hand extending dorsally. Carpal tunnel syndrome was diagnosed according to clinical examination and analysis. Giant lipoma causing carpal tunnel syndrome is particularly rare.
\end{abstract}

\section{Introduction}

Carpal tunnel syndrome is the most common entrapment neuropathy of the upper extremity. Soft tissue tumors that cause compression are rare. Lipomas constitute about $5 \%$ of the benign neoplasms of the hand, and thus they are the most common cause of compression related carpal tunnel syndromes (Bagatur and Yalçınkaya, 2009).

\section{Case Report}

A 65-years-old female patient presented with numbness and paresthesia in the $1^{\text {st }}, 2^{\text {nd }}, 3^{\text {rd }}$ and radial half of the $4^{\text {th }}$ finger and weakness on the left hand which gradually increased. Her medical history revealed that she had been operated for a soft tissue tumor about 20 years ago and it apparently recurred, displaying an accelerated growth during the past three years (Fig. 1,2). She had no remarkable medical conditions except for hypertension. Physical examination revealed a large soft tissue mass starting from palmar surface of the hand extending dorsally. She displayed positive Phalen test and Tinel sign, and the grip strength of the left hand was decreased in comparison with the other hand. Direct radiographs of the hand displayed only soft tissue edema and magnetic resonance imaging (MRI) revealed a soft tissue mass consistent with a lipoma.
Carpal tunnel syndrome is confirmed by electromyography (EMG). The surgical procedure was performed under axillary nerve block and tourniquet control. The soft tissue mass which occupied the midpalmar space and extended to the dorsal compartment of the hand through between the $3^{\text {rd }}$ and $4^{\text {th }}$ metacarpals was accessed via a palmar incision extending from the wrist to the third/fourth web space and totally removed by fine dissection (Fig. 3). The specimen was well demarcated, lobulated, yellow-brown in color and 7x7 $3 \mathrm{~cm}$ in size (Fig. 4). The incision was primarly sutured following bleeding control after tourniquet release.

The patient was discharged after two days without any early or late complications. The histopathological analyses confirmed the preliminary diagnosis of lipoma. The patient's pain and numbness resolved in the early postoperative period and grip strength fully recovered within two months postoperatively (Fig. 5).

\section{Discussion}

Carpal tunnel syndrome is the most common entrapment neuropathy of the upper extremity. The reason of the carpaltunnel syndrome is mostly unknown, but rarely soft tissue masses may cause it by compressing the median nerve (Nakamichi 


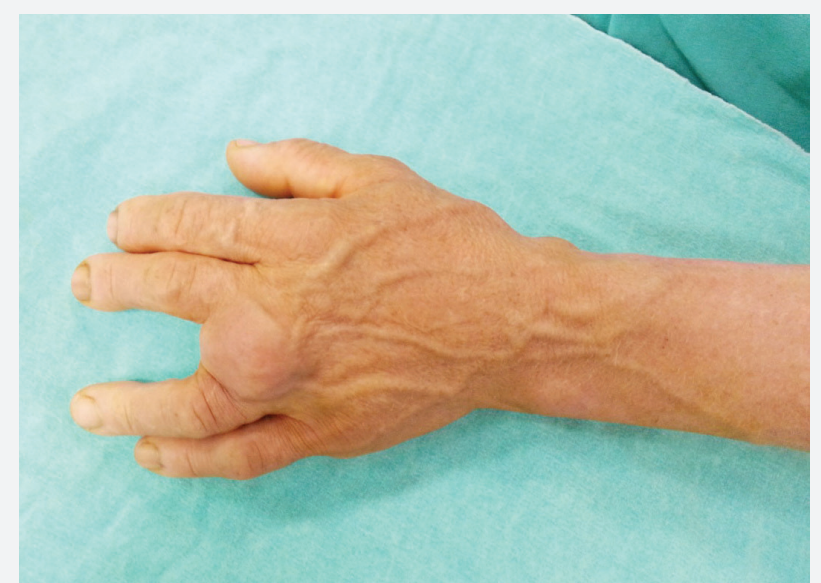

Fig. 1. Giant lipoma of hand.

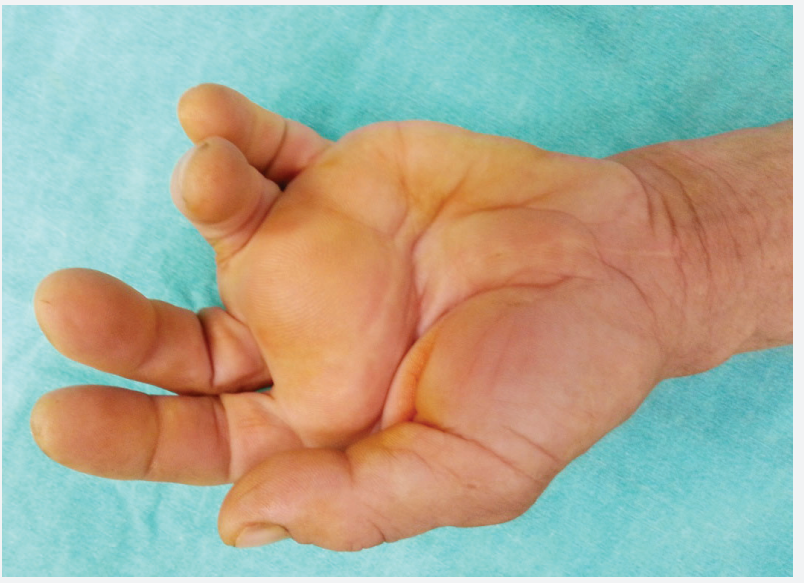

Fig. 2. Giant lipoma of hand.

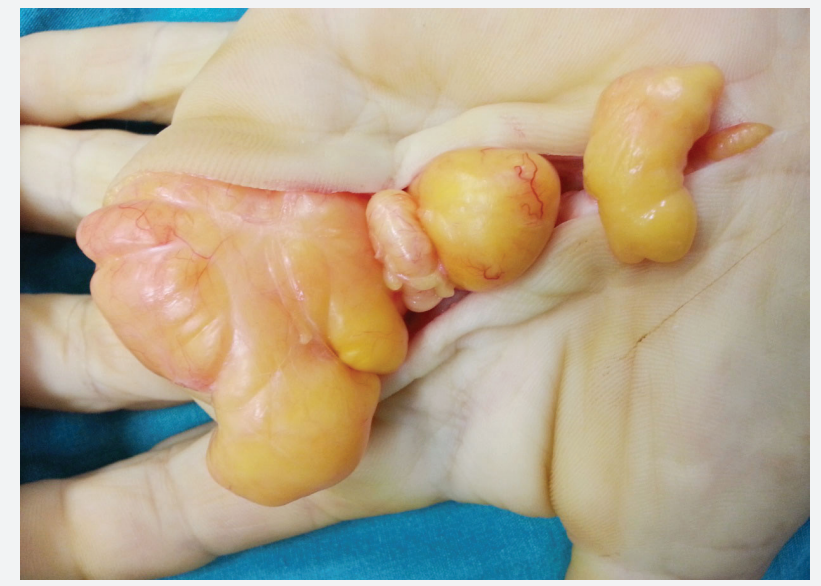

Fig. 3. Intraoperative image of tumour.

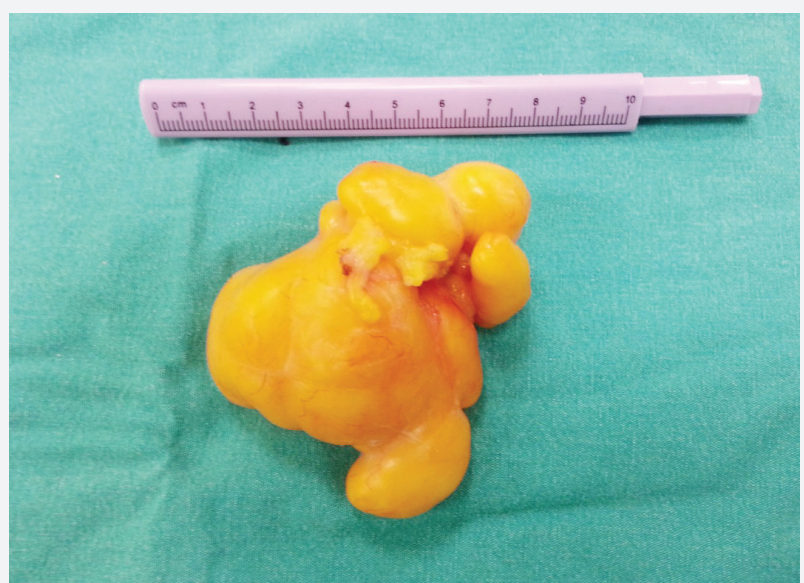

Fig. 4. The soft tissue mass of $7 \times 7 \times 7 \mathrm{~cm}$ in size after removed.

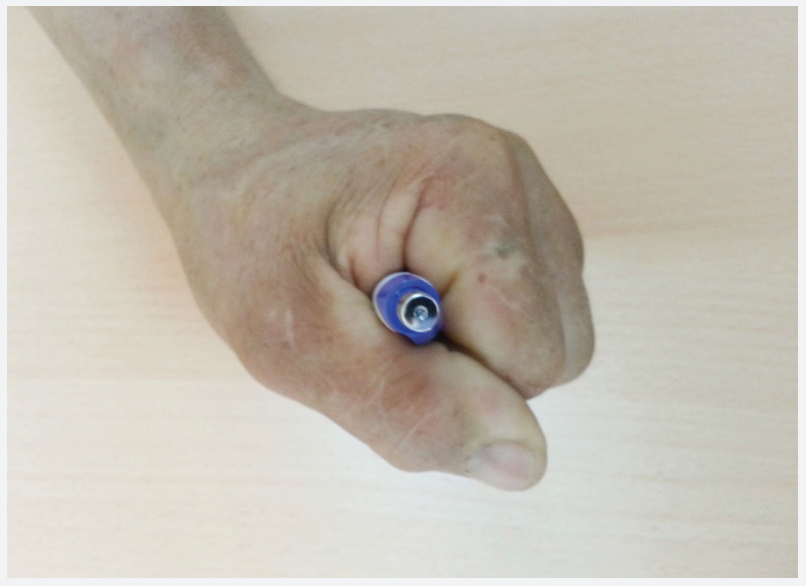

Fig. 5. Patient's grip strength fully recovered after two months.

and Tachibana, 1993). Lipomas are delicately encapsulated benign lesions, and MRI is the most reliable tool for their diagnosis, as well as reckoning their size and projections (Horch et al., 1997). The treatment is simply surgical removal (Inaparthy and Southgate, 2006). Lipomas are unlikely to recur after excision. Then again, the surgeon needs to be vary for prevention of recurrence and treatment of complications such as infection, nerve injury and soft tissue problems.

Although lipomas are benign soft tissue tumors, they can lead to carpal tunnel syndrome, especially when they are present in the midpalmar region of the hand by causing mechanical compression. The surgical excision needs to be planned via careful physical examination and correct utilization of the diagnostic tools such as MRI, in order to fully understand the surgical anatomy involved.

\section{REFERENCES}

Bagatur, A.E., Yalcinkaya, M., 2009. Unilateral carpal tunnel syndrome caused by an occult palmar lipoma. Orthopedics. 32, 18-20.

Horch, R.E., Allmann, K.H., Laubenberger, J., Langer, M., Stark, G.B., 1997. Median nerve compression can be detected by magnetic resonance imaging of the carpal tunnel. Neurosurgery. 41, 76-78.

Inaparthy, P., Southgate, G.W., 2006. Giant lipoma of hand: A case report. EJOST. 16, 251-252.

Nakamichi, K., Tachibana, S., 1993. Unilateral carpal tunnel syndrome and space-occupying lesions. J. Hand Surg. Br. 18, 748-749. 